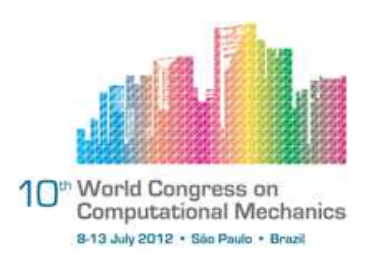

\title{
A HYBRID TWO-LEVEL APPROACH USED IN THE OPTIMIZATIONA OF AN AERONAUTICAL COMPOSITE STRUCTURE
}

\author{
L. D. Chiwiacowsky ${ }^{1}$, P. Gasbarri ${ }^{2}$, A. T. Gómez ${ }^{1}$, H. F. Campos Velho ${ }^{3}$, R. Monti ${ }^{2}$ \\ ${ }^{1}$ Master Program in Applied Computing (PIPCA), University of the Sinos Valley (UNISI- \\ NOS), Brazil (ldchiwiacowsky; breno@unisinos.br) \\ ${ }^{2}$ Department of Mechanical and Aerospace Engineering, Sapienza University of Rome, Italy \\ (paolo.gasbarri; riccardo.monti@uniroma1.it) \\ ${ }^{3}$ Associate Laboratory of Computing and Applied Mathematics (LAC), National Institute for \\ Space Research (INPE), Brazil (haroldo@lac.inpe.br)
}

\begin{abstract}
Nowadays composite materials are becoming increasingly popular, due to their ability to improve the structural performance and also to be tailored to meet specific design requirements for a given application. In the case of a wing composite structure, this is composed of a large number of panels, which have to be designed simultaneously to obtain an optimum structural design. In general, the wing-box design process is a multidisciplinary one, involving couplings and interactions between several disciplines such as aerodynamics, structural analysis, dynamics, and aeroelasticity. Therefore, the development of multidisciplinary design optimization (MDO) techniques, in which different disciplines and design parameters are coupled into a closed loop numerical procedure, seems appropriate to face such a complex optimization problem, such as a multilevel approach. The aeroelastic optimization here presented is relevant to the determination of the orientation of different layers which constitute the composite panels of a wing structure, that realizes the maximum flutter speed. By using a multilevel approach, the aeroelastic optimization problem can be decomposed into one subproblem, affecting the global response of the wing, and several independent subproblems, affecting portions of the wing. In the first level, the anisotropy parameters will be defined by a real coded Genetic Algorithm (GA), while at the second level of the optimization process, the ply orientation for the laminate composite plates will be defined by another Genetic Algorithm, with an integer encoding. For each one of the GAs, a local search procedure heuristic is applied to improve the best solution found by the GA. The hybrid strategy is shown to be efficient in maximizing the value of flutter velocity.
\end{abstract}

Keywords: Structural optimization, Multilevel optimization, Composite structures, Genetic algorithm, Local Search. 


\section{INTRODUCTION}

A wing composite structure is composed of a large number of panels, which have to be designed simultaneously to obtain an optimum structural design. Usually the design of each panel requires a large number of design variables to describe its geometry, ply composition and laminate stacking sequences. By virtues of these considerations, the design of all the panels of the wing-box simultaneously constitutes an extremely large optimization problem. It also requires a very detailed structural model of the entire wing, that appears to be beyond the present computational capabilities. In general the wing-box design process is a multidisciplinary one, involving couplings and interactions between several disciplines such as aerodynamics, structural analysis, dynamics, and aeroelasticity. The optimization problem, related to a wing-box design, involves multiple objectives and constraints pertaining to the design criteria associated with each of these disciplines.

These couplings and interactions can be exploited by an optimization procedure if all the disciplines are accounted for simultaneously rather than sequentially [17]. Therefore, the development of multidisciplinary design optimization (MDO) techniques, in which different disciplines and design parameters are coupled into a closed loop numerical procedure, seems appropriate to face such a complex optimization problem. With a multilevel decomposition approach [15, 18], a large complex optimization problem is broken into a hierarchy of smaller optimizations subproblems, as shown in Figure 1 (from reference [17]).

The aeroelastic optimization here presented is relevant to the determination of the orientation of different layers which constitute the composite panels of a wing-box structure, that realizes the maximum flutter speed. By using a multilevel approach the aeroelastic optimization problem can be decomposed into one subproblem, affecting the global response of the wing, and several independent subproblems, affecting portions of the wing. For the case under concern the flutter velocity is used to describe the global response of the wing. Therefore a two level decomposed wing optimization problem can be defined as shown in Figure 2.

The upper level optimizes the wing by changing global quantities, here defined through some parameters related to the degree of anisotropy of the material. These parameters are treated as independent quantities even if they are not. The reconciliation between these quan-

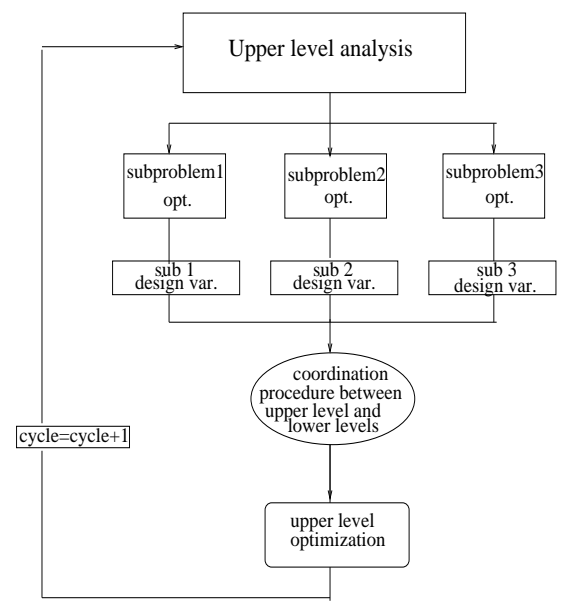

Figure 1. General multilevel optimization procedure with two levels. 


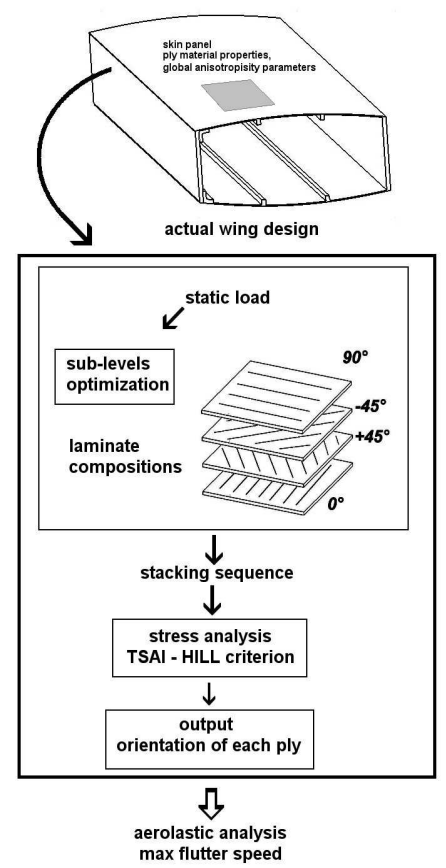

Figure 2. Wing-box optimization criterion.

tities and the real design variables is done on the lower level. The lower level consists of independent subproblems at different stations along the wing, which optimize the lay-up angles to reconcile the upper level independent variables. The coupling between the upper level problems and the lower ones is preserved through a coordination procedure such as that described in [14].

Therefore, the application of a two-level method to characterize composite panels of a wing-box structure is discussed. In the first level, the anisotropy parameters will be defined by a real coded Genetic Algorithm [10], while at the second level of the optimization process, the ply orientation for the laminate composite plates will be defined by another Genetic Algorithm, with the integer encoding [6]. In both optimization levels a local search heuristic is used coupled with the GA. Although GA can rapidly locate the region in which the global optimum exists, they usually take a relatively long time to locate the exact local optimum in the region of convergence [16]. A combination of a GA and a local search method can speed up the search to locate the exact global optimum. In this work the Hooke-Jeeves method [8] is employed as an intensification heuristic in order both to reduce the time needed to reach the global (or local) optimum and to provide a better solution if compared with that provided by the GA metaheuristic when employed as a single optimization method.

The same problem was recently solved successfully thanks to a hybrid multilevel approach [5] where a deterministic method is used in the first level optimization and a stochastic method is used in the second level optimization. In this previous approach, the local search method was not employed and the present work presents a new strategy, based only on Genetic Algorithms coupled to a local search heuristic, which is shown to be more efficient in maximizing the value of flutter velocity. 


\section{WING STRUCTURE}

In this work the wing structure consists of a wing-box stiffened composite structure $[13,4]$. A plate model is used to represent the wing-box. The planform geometry of this plate is assumed to be trapezoidal, with an arbitrary sweep angle $\Lambda$, with respect to the wind direction, and a non dimensional non-orthogonal coordinate system $(u, \eta)$ are also adopted as reported in equation (1).

$$
\left\{\begin{array}{l}
\eta(X, Y)=Y / L, \quad 0 \leq \eta \leq 1 \\
u(X, Y)=-\frac{1}{2}+\frac{X-\tan \Lambda Y}{c}, \quad-1 / 2 \leq u \leq 1 / 2 .
\end{array}\right.
$$

The wing here considered has a uniform cross section. The presence of ribs, ensuring the transfer of shear stress between the upper and lower skins of the wing-box is assumed in order to neglect the shear deformations of the cross-sections. The upper and lower skins are made of several layers that are assumed to have the same mechanical properties with different orientations. Furthermore, the upper and lower skins and the corresponding layers are assumed to be symmetric about the middle plane of the wing. The anisotropic plate bending stiffness matrix $\mathrm{D}$ is evaluated by means of the classical laminated theory.

A modified finite element discretization (FEM), based on the following kinematics representation to describe the flexural displacement of the wing-box, was assumed [13]:

$$
w(u, \eta)=w_{0}(\eta)+u w_{1}(\eta)+u^{2} w_{2}(\eta)+\ldots+u^{N} w_{N}(\eta) .
$$

For the sake of brevity all the analytical details, relevant to this methodology and its use to evaluate (via the classical FEM theory) the mass matrix, the stiffness matrix and consequently the dynamic behavior of a composite swept wing, are omitted and can be found in [13]. Suffice here to say that the final form is as follows:

$$
\mathbf{M} \ddot{\mathbf{X}}+\mathbf{K X}=\mathbf{F}_{e x t},
$$

where $\mathbf{M}$ and $\mathbf{K}$ are the mass matrix and the stiffness matrix of the structure and $\mathbf{F}_{\text {ext }}$ is the vector of the external forces.

\subsection{Aerodynamic model}

For the present study it is necessary to have an aerodynamic tool that can adequately describe the aerodynamic unsteady loads acting on the wing. One of the most popular methods that can be used to determine aerodynamic forces in subsonic compressible flow is the Kernel Function Method (KFM), which is able to characterize the aerodynamic pressure in some wing collocated points. The equation of KFM here considered is the one of quasi steady aerodynamics:

$$
\frac{w(x, y)}{U_{\infty}}=\frac{1}{8 \pi q_{\infty}} \oint_{\Sigma} G\left(x, y, \bar{x}, \bar{y} ; M_{\infty}\right) \Delta p(\bar{x}, \bar{y}) d \Sigma,
$$

where $(x, y)$ and $(\bar{x}, \bar{y})$ are the coordinates of a generic control point and of the doublet singularity point, respectively, and $\oint$ here denotes Hadamard's principal value of the integral. 
The idea of the KFM is to approximate the unknown pressure distribution $\Delta p(\bar{x}, \bar{y})$ in chordwise and span-wise directions by prescribed functions with unknown coefficients. The final expression for the pressure distribution reads:

$$
\Delta \bar{p}(u, \eta)=8 \pi q_{\infty} \boldsymbol{\Gamma}^{T}(u, \eta) \mathbf{A}^{-1} \mathbf{J},
$$

where $\Gamma^{T}(u, \eta)$ is the aerodynamic shape function vector, $\mathbf{A}^{-1}$ is the inverse matrix of the algebraic solving system (depending on Mach number $M_{\infty}$ ), and $\mathbf{J}$ is the vector of downwashes evaluated at the aerodynamic control points $P_{m n}$ [12].

\subsection{Aeroelastic equations}

The vector form of the aerodynamic forces acting on the wing-box to be used with FEM formulation reads as follows:

$$
\mathbf{F}_{\mathrm{ext}}=q_{\infty} \mathbf{R} \mathbf{J}
$$

being $\mathbf{R}$ the so-called aerodynamic matrix. The vector of down-washes $\mathbf{J}$ can be divided into two parts, the one relevant to geometric incidence $\alpha_{g}$ and the one relevant to elastic incidence $\alpha_{e}[2]:$

$$
\mathbf{J}=\alpha_{g}+\alpha_{e} .
$$

The elastic incidence can be expressed through the deflection of the structural points of finite element discretization: $\alpha_{e}=\mathrm{QX}$, being $\mathrm{Q}$ the matrix which transforms the structural degrees of freedom into the aerodynamic ones. Combining equations (6) and (7) and substituting them into equation (3) the final aeroelastic system is obtained:

$$
\mathbf{M} \ddot{\mathbf{X}}+\mathbf{K X}=q_{\infty} \mathbf{R}\left\{\alpha_{g}+\mathbf{Q X}\right\}=\mathbf{F}_{a e r} .
$$

The above equation is the basic formula for studying the aeroelastic phenomena (both static and dynamic) on elastic wings.

The flutter analysis problem will be solved, at first level, by expressing stiffness, mass and aerodynamic forces in the smaller subspace. The equations of motion (8) are transformed into frequency domain resulting in a non linear eigenvalue problem to be solved for a given flight condition [13].

\section{WING-BOX OPTIMIZATION}

\subsection{Composite laminate}

The composite laminate design process typically involves the optimization of the following three parameters: $i$ ) Ply (or lamina) material; ii) Ply thickness and iii) Ply orientation.

In this paper only the optimal orientation problem of a two-dimensional linearly elastic structure, characterized by the following constitutive law, has been considered ${ }^{1}$ :

$$
\left\{\begin{array}{l}
\sigma_{1} \\
\sigma_{2} \\
\tau_{12}
\end{array}\right\}=\left[\begin{array}{lll}
H_{11} & H_{12} & H_{16} \\
H_{21} & H_{22} & H_{26} \\
H_{31} & H_{32} & H_{36}
\end{array}\right]\left\{\begin{array}{l}
\epsilon_{1} \\
\epsilon_{2} \\
\gamma_{12}
\end{array}\right\}=[\mathbf{H}]_{j}\left\{\begin{array}{l}
\epsilon_{1} \\
\epsilon_{2} \\
\gamma_{12}
\end{array}\right\},
$$

\footnotetext{
${ }^{1}$ Note that this constitutive law will be used for calculating the stiffness matrix $\mathbf{K}$ of the wing-box structure.
} 
where $\sigma_{1}, \sigma_{2}, \tau_{12}$ are stress components, $\epsilon_{1}, \epsilon_{2}$ and $\gamma_{12}$ are strain components. The $\mathbf{H}_{j}$ is the rotated orthotropic stiffness matrix for the $j$-th lamina of the composite laminate, which is computed by using the standard rotation matrix, with the final form as follows:

$$
\mathbf{H}_{j}=\mathbf{H}^{00}+\mathbf{H}^{01} \cos 2 \theta_{j}+\mathbf{H}^{02} \cos 4 \theta_{j}+\mathbf{H}^{10} \sin 2 \theta_{j}+\mathbf{H}^{12} \sin 4 \theta_{j},
$$

where the matrix $\mathbf{H}^{00}$ and the matrices $\mathbf{H}^{l m}(l=0,1$ and $m=0,1,2)$ contain the invariants w.r.t the rotation operation, e.g terms that are not dependent on ply orientation.

From the expression of the matrix $\mathbf{H}_{j}$ (see equation (10)) it is possible to observe that its coefficients are harmonic functions of the orientation angle $\theta$. This is a complicating factor for the optimization process of the composite laminate structure, since design functions have revealed the presence of multiple optima [7].

\subsection{Laminate plate theory: local variables vs. global variables}

In this work the case of symmetric orthotropic laminate is considered, with the orthotropic plate bending stiffness matrix $[\mathbf{D}]$ defined as follows:

$$
\mathbf{D}=\sum_{j=1}^{N_{L a}} \frac{\bar{z}_{j}^{3}-\bar{z}_{j-1}^{3}}{3} \mathbf{H}_{j},
$$

where $\bar{z}_{j}=\left(z_{j}+z_{j-1}\right) / 2$ is the position of the $j$-th layer and $N_{L a}$ is the number of layers.

By substituting expression (10) into equation (11) the matrix of bending stiffness $\mathbf{D}$ is obtained as follows:

$$
\mathbf{D}=\mathbf{D}^{00}+\mathbf{H}^{01} \zeta_{3}+\mathbf{H}^{02} \zeta_{1}+\mathbf{H}^{10} \zeta_{4}+\mathbf{H}^{12} \zeta_{2},
$$

where the terms $\zeta_{i}(i=1, \ldots, 4)$ are functions of the orientation of the layers of laminate, of their thickness and of their stacking sequence, such that the expression $\mathrm{D}^{00}$ and the functions $\zeta_{i}$ are defined as:

$$
\begin{gathered}
\mathbf{D}^{00}=\sum_{j=1}^{N_{L a}} \frac{z_{j}^{3}-z_{j-1}^{3}}{3} \mathbf{H}^{00} ; \\
\zeta_{1}=\sum_{j=1}^{N_{L a}} \frac{z_{j}^{3}-z_{j-1}^{3}}{3} \cos 4 \theta_{j} ; \quad \zeta_{2}=\sum_{j=1}^{N_{L a}} \frac{z_{j}^{3}-z_{j-1}^{3}}{3} \sin 4 \theta_{j} ; \\
\zeta_{3}=\sum_{j=1}^{N_{L a}} \frac{z_{j}^{3}-z_{j-1}^{3}}{3} \cos 2 \theta_{j} ; \quad \zeta_{4}=\sum_{j=1}^{N_{L a}} \frac{z_{j}^{3}-z_{j-1}^{3}}{3} \sin 2 \theta_{j} .
\end{gathered}
$$

As mentioned before, the goal of this optimization investigation is to improve the aeroelastic performance of an airplane wing by using composite tailoring and determining the optimal orientation of the composite laminates. For the case under concern the local design variables of the optimization are the $\theta_{j}\left(j=1, \ldots, N_{L a}\right)$, whose values must be determined in order to maximize the aerodynamic pressure $q_{\infty}$ undergoing certain composite failure constraint conditions. In the present optimization procedure (first level) the orientation of the layers are substituted by their resulting trigonometric combinations $\zeta_{i}(i=1, \ldots, 4)$ which describe now the degree of the anisotropy of the composite structure and represent global design variables. 


\subsection{First Level Optimization}

The first level optimization consists of finding the maximum value of flutter velocity for assigned values of global design variables $\zeta_{i}$ along the wingspan. The formulation of the problem may be stated as follows:

Find $(\mathcal{X})$ to maximize $\mathcal{F}_{I}(\mathcal{X})$

subject to

$$
\begin{aligned}
& g_{k}(\mathcal{X}) \leq 0 ; \text { and } h_{k}(\mathcal{X})=0 \\
& k=1, \ldots, N_{L} ; \quad \mathcal{X}_{l} \leq \mathcal{X} \leq \mathcal{X}_{u}
\end{aligned}
$$

where $\mathcal{F}_{I}(\mathcal{X})$ is the flutter velocity obtained from the solution of the frequency domain eigenvalue problem derived from equation (8), $\mathcal{X}$ is the design variable vector, containing the global design variables in an assigned number $N_{L}$ of wingspan locations; the subscripts $l$ and $u$ represent lower and upper limit on design variables respectively, and $g_{k}$ and $h_{k}$ are inequality and equality constraints respectively.

\subsection{Second Level Optimization: from global variables to local variables}

The second level optimization is composed by $N_{L}$ subproblems which are to be solved for different portions of wingspan. The purpose of each lower level optimization is to identify the ply orientation angles which are used in the composite panels building, for each assumed wingspan location. The computed orientation angles have to provide a configuration which yield the stiffness imposed by the solution of the first level optimization problem, i.e., provide a composite panel configuration which is able to maintain the strength to withstand loads calculated by the upper level analysis. The lower level design variables are integer values for the orientation angles $\theta_{j} \in\left[-90^{\circ},+90^{\circ}\right]\left(j=1, \ldots, N_{L a}\right)$ of each ply of the laminate. The objective function is defined as the difference between $\bar{\zeta}_{i}(i=1, \ldots, 4)$, which are imposed by the first level optimization, and the $\zeta_{i}$ 's which are computed based on the second level optimization design variables $\theta_{j}$, as defined in equation (14):

$$
\mathcal{F}_{I I}=\frac{\left(\zeta_{1}-\bar{\zeta}_{1}\right)^{2}+\left(\zeta_{2}-\bar{\zeta}_{2}\right)^{2}+\left(\zeta_{3}-\bar{\zeta}_{3}\right)^{2}+\left(\zeta_{4}-\bar{\zeta}_{4}\right)^{2}}{\bar{\zeta}_{1}^{2}+\bar{\zeta}_{2}^{2}+\bar{\zeta}_{3}^{2}+\bar{\zeta}_{4}^{2}},
$$

where barred quantities denote upper level design variables.

After the minimization of the objective function (15) the process returns to the upper level for the next optimization cycle. Note that the number of second level optimizations is equal to the number $N_{L}$ of wingspan sections.

\subsection{Coordination between upper and lower levels}

The coordination between first and second levels is implemented by first level constraints. Specifically, these constraints, one per each second level optimization, have been implemented in two different ways; the first one consists of imposing the following equality constraints condition:

$$
h_{k}=\mathcal{F}_{I I, k}^{L}=0 ; \quad k=1, \ldots, N_{L},
$$


where $\mathcal{F}_{I I, k}^{L}$ is the most recent value of the $k$-th second level objective function, and $N_{L}$ is the number of second level problems. The second type of constraint is inherent to the strength of the composite panel, and it can be imposed by using the classical composite failure criteria such as the Tsai-Hill criteria [1]. This constraint is evaluated at the upper layer of the laminate and has the following form:

$$
g_{k}=\left(\frac{\sigma_{1}}{\sigma_{1}^{*}}\right)^{2}-\left(\frac{\sigma_{2}^{*}}{\sigma_{1}^{*}}\right)\left(\frac{\sigma_{1} \sigma_{2}}{\sigma_{1}^{*} \sigma_{2}^{*}}\right)+\left(\frac{\sigma_{2}}{\sigma_{2}^{*}}\right)^{2}+\left(\frac{\tau_{12}}{\tau_{12}^{*}}\right)^{2}-1 \leq 0,
$$

with $k=1, \ldots, N_{L}$, where starred quantities $\sigma_{1}^{*}, \sigma_{2}^{*}$ and $\tau_{12}^{*}$ denote the strength values along the Principal Material Directions, whereas no starred quantities are the stresses associated to an external load.

\section{OPTIMIZATION AND NUMERICAL RESULTS}

Two different Genetic Algorithm (GA) methods, within a two-level strategy, were applied to design a wing-box structure in order to maximize the value of flutter velocity. One of the GA methods was employed for the solution of the optimization problem at the upper level, i.e. find the maximum flutter velocity, assuming the anisotropy parameters $\zeta_{k}$ $(k=1, \ldots, 4)$ as design variables. On the other hand, another GA method was used to solve the different optimization problems at a the lower level related in the finding of the orientation angles $\theta_{j}(j=1, \ldots, N)$ for the laminate composite sequence in some prescribed wingspan locations.

As a class of general-purpose search methods, the GA metaheuristic give a remarkable balance between the exploitation of the promising regions of the search space and the exploration of the search space. They differ from more conventional optimization techniques since they work on the whole populations of encoded solutions, called chromosomes or individuals, and each possible solution is encoded as a set of genes.

In general, the most important phases in standard GAs are selection (competition), reproduction, mutation, and fitness evaluation. Selection is an operation used to decide which individuals to use for the reproduction in order to produce new search points. Reproduction or crossover is the process by which the genetic material from two parent individuals is combined to obtain one or more offsprings. Mutation is normally applied to one individual in order to produce a new version of it where some of the original genetic materials have been randomly changed. Fitness evaluation is the step where the quality of an individual is assessed [6]. Beyond the standard genetic operators described before, another operator called Epidemical [3] was used. This operator is activated when a specific number of generations is reached without any improvement of the best individual. When it is activated, all the individuals in the population are replaced, except the fittest individuals (e.g., first $2 \%$ with the best fit in the population). This strategy is used to introduce new genetic characteristics in the population.

Furthermore, by conducting the search in a global domain, the GA metaheuristic reduces the chance of converging to local optima and makes it possible to solve problems involving many parameters. Other advantages in the usage of GA are that it is a self-start method with no special requirement on the initial value of unknown parameters, other than 
the definition of a search range, and also it does not need any information such as gradients or derivatives of the function to be optimized.

\subsection{The upper level GA}

At the upper level of the two-level optimization procedure, the anisotropy parameters $\zeta_{k}(k=1, \ldots, 4)$ are sought in order to maximize the value of flutter velocity, and also to satisfy both the equality and inequality constraints. This constrained optimization problem can be converted into an equivalent unconstrained problem by using the constraints as penalty functions in a modified objective function, as described in [7]. Thus, the maximization problem shown in section 3.3 is transformed into a minimization problem, and it is solved by a GA assuming the fitness function defined as follows:

$$
\phi_{I}=w_{v} \frac{1}{V_{f}}+\sum_{k=1}^{N_{L}} w_{g} \max \left(0, g_{k}\right)+\sum_{k=1}^{N_{L}} w_{h} \max \left(0, h_{k}\right),
$$

where $V_{f}$ is the value of the flutter velocity; $g_{k}$ and $h_{k}$ represent the inequality and equality constraints, as defined in equations (16) and (17), respectively. The constants $w_{g}$ and $w_{h}$ are the penalty parameters associated with the inequality and equality constraints, which penalize infeasible solutions. On the other hand, the parameter $w_{v}$ is used in order to balance the importance of the flutter velocity in the fitness function. The values assumed for each parameter were chosen empirically, by performing some numerical analysis in advance.

Since the upper level design variables are represented by real numbers, which are used for encoding the individuals for the GA, the appropriate genetic operators employed here are: Tournament Selection [11, 10], Arithmetic Crossover [10] and Non-uniform Mutation [10]. For the minimization procedure, based on the fitness function $\phi_{I}$, equation (18), it has been set a population size of 30 individuals, $40 \%$ mutation rate, $100 \%$ crossover rate and as stopping criteria the maximum number of 500 generations.

\subsection{The lower level GA}

At the lower level, a second Genetic Algorithm is proposed in order to solve the minimization problem stated in section 3.4. The orientation angles $\theta$ for the composite laminate are to be determined basing on the values $\zeta_{k}$ imposed by the upper level optimization. The stacking sequence consists of six sub-groups of layers $\left(\Theta=\left[\theta_{1} / \theta_{2} / \theta_{3} / \theta_{4} / \theta_{5} / \theta_{6}\right]\right)$ where each sub-group $\theta_{j}(j=1, \ldots, 6)$ consists in turn of four layers oriented in the same way. This sequence of angles was mapped as integer values which were used for encoding the individuals for the GA. Thus each integer value represents a gene in the total individual. The objective function defined in equation (15) is the fitness function used by the GA in the minimization process, which employs the following genetic operators: Tournament Selection [11, 10], Two-point Crossover [6], Uniform Mutation [10] and Epidemical [3]. For the numerical simulations, a population size of 50 individuals, $10 \%$ mutation rate, $100 \%$ crossover rate and Epidemical activated after 300 consecutive generations without improvement of the best individual have been set. Moreover, for numerical reasons, the equality constraint defined by equation (16) can be relaxed so that the optimization procedure stops after a number of 500 consecutive generations without any improvements of the fittest solution. 


\subsection{The hybrid approach}

The two-level optimization procedure, here proposed to optimize the aeroelastic behavior of a wing-box structure, could be defined as a hybrid approach since the optimization problems of each level are solved by two different GA metaheuristics. Furthermore, for each optimization level, the GA metaheuristic was applied coupled with a local search heuristic, the Hooke-Jeeves method [8]. The GA works with a population of solutions that enables a wide search on the space of solution (diversification), mainly by using mutation operators adequately. However, GA often uses local search techniques for intensification, in order to improve some solutions of the population until a local optimum is reached. By using local search schemes, the search should be speeded up since domain specific knowledge is used, providing better quality solutions if compared with that solutions provided without the application of a intensification strategy [19].

The GA is defined as population-based metaheuristic while the Hooke-Jeeves (HJ) heuristic, here used as local search method, is defined as a direct search algorithm which is a class of search algorithms that avails itself to the solution of optimization problems. This method evaluate the objective function at sample points and use the provided information to continue sampling along promising directions. A prerequisite is an initial point inside the feasible region as well as a set of search directions. The HJ method works similarly to coordinate descent, however it is a more aggressive search. In addition to the feasible initial solution, also a set of search directions $\mathbf{v}$ is provided as well as an array $\mathbf{s}$ of $M$ scales, that determine the step length size which the search algorithm is allowed to explore from the current point when investigating for promising search directions. In the following, the pseudocode of HJ method is presented, assuming the design variable vector as $\mathbf{x} \in \Re^{N}$, the objective function as $f(\cdot)$, and, without loss of generality, the optimization problem as one of minimizing the objective function [9]:

- Define: initial position $x_{0}$, search directions $\mathbf{v}$, and scales $\mathbf{s}$.

- Search:

1. Compute $f\left(x_{0}\right)$.

2. Choose scale $s_{i}$ from $\mathbf{s}$.

3. Exploratory step:

3.1 Define $x_{s}=x_{0}$.

3.2 Investigate search direction $v_{j} \in \mathbf{v}$ :

- Compute $f\left(x_{s}+s_{i} v_{j}\right)$. If $f\left(x_{s}+s_{i} v_{j}\right)<f\left(x_{0}\right)$ define $x_{s}=x_{s}+s_{i} v_{j}$, move to step 3.3.

- If $f\left(x_{s}+s_{i} v_{j}\right) \geq f\left(x_{0}\right)$ compute $f\left(x_{s}-s_{i} v_{j}\right)$.

- If $f\left(x_{s}-s_{i} v_{j}\right)<f\left(x_{0}\right)$ define $x_{s}=x_{s}-s_{i} v_{j}$, continue to step 3.3.

3.3 Repeat step 3.2 for $j=j+1$. If $j>N$ (all $N$ search directions in $\mathbf{v}$ investigated) continue to step 3.4 .

3.4 Obtain promis ing direction $d_{i}=x_{s}-x_{0}$.

4. If $d_{i}=0$, then $i=i+1$ (reduce scale size to $s_{i+1}$. If $i>M$ (all $M$ scales in $\mathbf{s}$ investigate) terminate search iterations.

5. Pattern move step:

- Define $x_{c}=x_{0}+2 d_{i}$. Compute $f\left(x_{c}\right)$. 


$$
\text { - If } f\left(x_{c}\right)<f\left(x_{s}\right) \text {, set } x_{s}=x_{c} \text {. }
$$

6. Set $x_{0}=x_{s}$, and repeat step 1 .

- Optimal point, $x_{0}$, obtained.

The hybrid approach is defined by using the HJ heuristic inserted in the AG evolutionary process along the generations. The evolutionary process is interrupted in previous determined moments for the local search execution based on the HJ method. For the local search activation, some parameters should be set: the frequency of activation, which solution from the GA population will be assumed as the initial solution for $\mathrm{HJ}$ heuristic; and which solution in the GA population will be replaced by the solution returned by the HJ heuristic. These parameters define the local search application and depending on its definition, different configurations for the local search could be tested. In this work, the parameters were defined for each optimization level, with the following assumed values:

- Frequency of activation: after 200 generations in the first level GA, and after 300 generations in the second level GA;

- Initial solution: the HJ initial solution will be assumed as the best solution in the GA population, based on its fitness value;

- Replaced solution: the HJ returned solution will replace the worst solution in the GA population, based on its fitness value.

When finished the search performed by the HJ heuristic, the GA evolutionary process is resumed, from the generation it was interrupted for the local search performing, and, as expected, with a new improved solution in the population.

\subsection{Numerical Results}

The efficiency and robustness of the hybrid two-level optimization procedure, based on Genetic Algorithm metaheuristics and on Hooke-Jeeves heuristic, was evaluated by solving different design problems of a composite laminate of a wing-box according to the values of structural parameters such as angle variation $\triangle \theta$ and transverse stiffness $E_{22}$.

Concerning the wing-box structure, it was set a 24 ply of Carbon Reinforced/Epoxy composite material with the following structural properties: $E_{11}=65 \mathrm{GPa}, E_{22}=$ to be assumed, $\nu_{12}=0.31, \nu_{21}=0.31, G_{12}=6.9 \mathrm{Gpa}, \rho=1650 \mathrm{Kg} / \mathrm{m}^{3}$, Tensile strength $\sigma_{1}=527 \mathrm{MPa}$, Tensile strength $\sigma_{2}=490 \mathrm{MPa}$, Tensile strength $\tau_{12}=55 \mathrm{MPa}$, NPLY=24, stringers Young's modulus $E=72 \mathrm{GPa}$, stringers mass density $\rho=2800 \mathrm{Kg} / \mathrm{m}^{3}$ and width of stringers $l=3.5 \mathrm{~cm}$.

As far as the static analysis is concerned, it has been performed at the second level to calculate the strength of the composite wing-box, and it is based on: $i$ ) applying static aerodynamic loads at a free stream velocity equal to the $80 \%$ of the flutter velocity evaluated at the first level; $i i$ ) considering the geometric incidence $\alpha_{g}=5^{\circ}$ constant along the wingspan and iii) considering the Tsai-Hill criterion applied at the upper ply of the wing-panels as a strength constraint for the composite panel. As far as the first level optimization procedure is concerned, the following parameters were assumed: $w_{v}=200, w_{g}=0.01$ and $w_{h}=0.1$.

The hybrid two-level optimization scheme was applied for a swept wing of $\Lambda=30^{\circ}$ and aspect ratio $\lambda=L / c=7$, where $L$ is the $Y$-wise semi-span, and $c$ is the chord, here considered as a constant along the wingspan. The wing-box height is here $h=14 \mathrm{~cm}$ whereas 


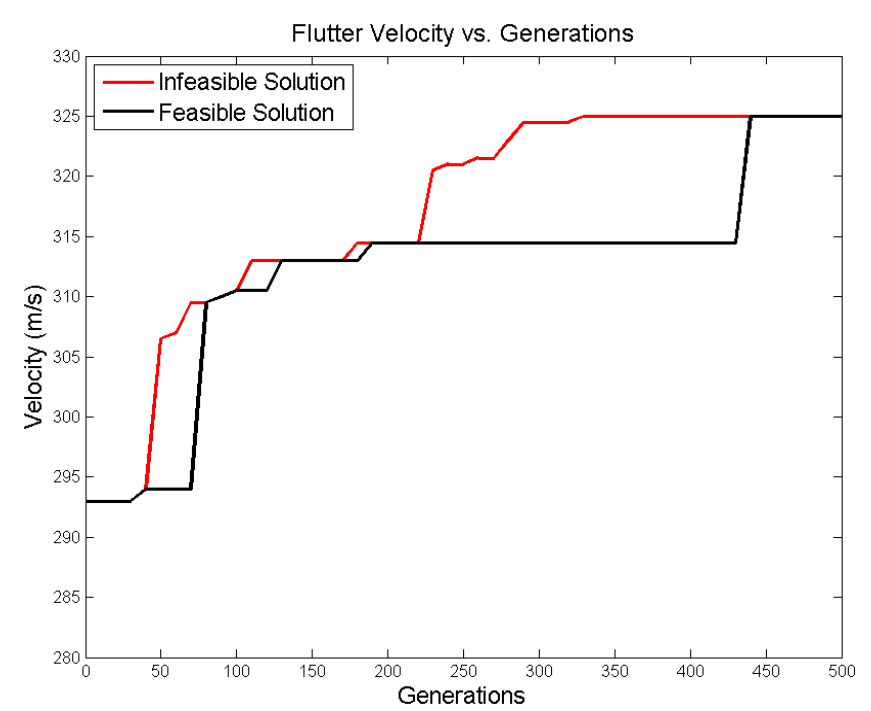

Figure 3. Flutter velocity [m/s] vs. generations, $\triangle \theta=1^{\circ}, E_{22}=65 \mathrm{GPa}$.

the thickness of the composite laminate is $h_{l}=4 \mathrm{~mm}$ and the width of the stringers is $a=3.5$ $\mathrm{cm}$. The wing-box is divided into five equal parts along the wingspan. Each part is here considered as a subproblem of the second level optimization procedure as shown in Figure 1.

As far as the second level optimization procedure is concerned and the determination of the angles $\theta_{j}$ of the stacking sequences $\Theta$, as mentioned before, different situations were analyzed according to different choices of the angle variation $\Delta \theta_{j}$ and transverse stiffness $E_{22}$. In particular, the results obtained by the combination of $\Delta \theta_{j}$ equals to $1^{\circ}, 5^{\circ}, 10^{\circ}, 20^{\circ}$, $30^{\circ}$ and $45^{\circ}$ and $E_{22}$ equals to $65 \mathrm{GPa}, 75 \mathrm{GPa}$ and $85 \mathrm{GPa}$ will be discussed.

Figure 3 shows the flutter velocity evolution according to the generation number of the upper level optimization, for both feasible and unfeasible solutions. At this optimization level, where a constrained optimization problem is to be solved, the GA is allowed to search for solutions also in the infeasible search space. As noted in $[7,10]$, the infeasible search opens new routes to optima and permits the algorithm to exploit short-cuts in the search space if any. It is also worth to note that, in general, a new feasible solution is obtained by the GA some generations after a better infeasible solution is provided. However, this behavior is weakly noted for angle variations $\triangle \theta=45^{\circ}$ because greater angle variations could imply the non existence of a lay-up sequence so that the Tsai-Hill failure criterion is not violated. Also in Figure 3, it is possible to observe that the flutter velocity strongly increases up to the half of its evolution period. After that, the improvements are scarce, which indicates a local optimum trap.

It is now of interest to analyze the effects of the transverse stiffness $E_{22}$ and angle variations $\Delta \theta_{j}$ used at the second level on the optimized flutter velocity. Figures 4 and 5 show the parametric study where the optimized flutter velocity is evaluated for different values of $\Delta \theta_{j}$ and $E_{22}$. The higher values for the flutter velocity were identified, for each configuration, from a set of 30 runs of the hybrid two-level optimization procedure. It should be pointed out that, for the same value of angle variation, as $E_{22}$ increases, the maximum value on the optimized flutter velocity $V_{f}$ also increases, as depicted in Figure 4. This behavior is easily explained since by increasing the mechanical properties of all the laminates also the flexural/torsional rigidity of the wing-box increases and hence the flutter velocity of the wing. Analogously, for the same transverse stiffness, as $\Delta \theta_{j}$ increases, the maximum value on the optimized flutter velocity $V_{f}$ decreases, as depicted in Figure 5. The reason for this behavior is due to the fact that for a smaller value of $\Delta \theta_{j}$ there is a larger number of possible choices in the range 


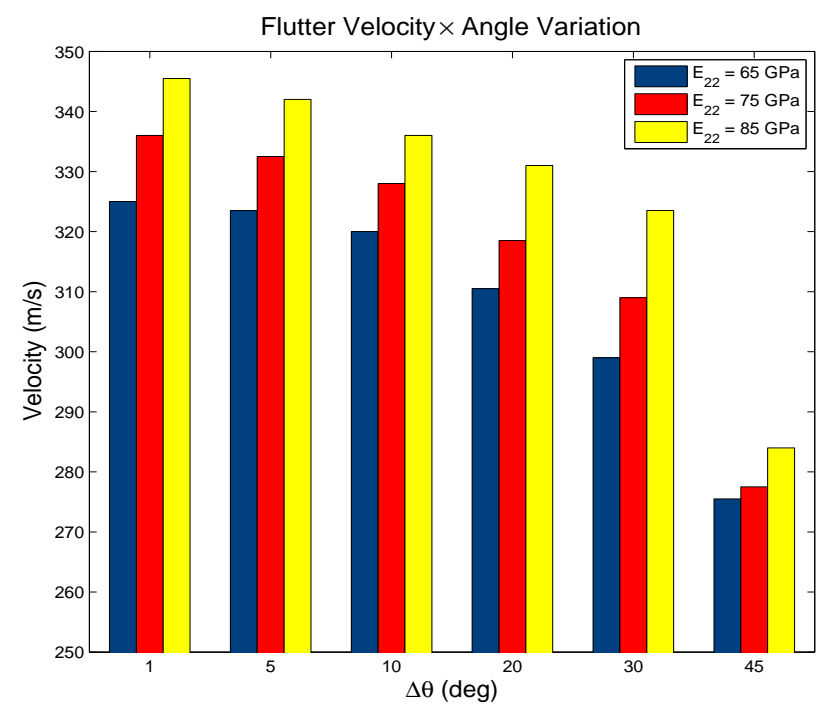

Figure 4. Flutter Velocities vs. Angle Variation for different transverse stiffness $E_{22}$.

$\left[-90^{\circ}, 90^{\circ}\right]$ made by the GA that makes it escape from local optima.

Concerning the maximum value of the optimized flutter velocity $V_{f}$, obtained with different angle variations $\Delta \theta$, a remarkable difference is observed between the results for $\Delta \theta_{j} \leq 30^{\circ}$ and $\Delta \theta_{j}=45^{\circ}$. This characteristic can be explained if we consider that, with a step variation of $45^{\circ}$, the search of the best lamination sequence performed in the second level $\mathrm{GA}$, has a small number of possible choices for each lamina, since the range $\left[-90^{\circ}, 90^{\circ}\right]$ was assumed. This small number of possible choices leads to a lay-up sequence so that the TsaiHill failure criterion is violated. Future works will be dedicated to the evaluation of different search strategies by changing the crossover operator, or even by changing the optimization method, for example, by using a trajectory-based metaheuristic.

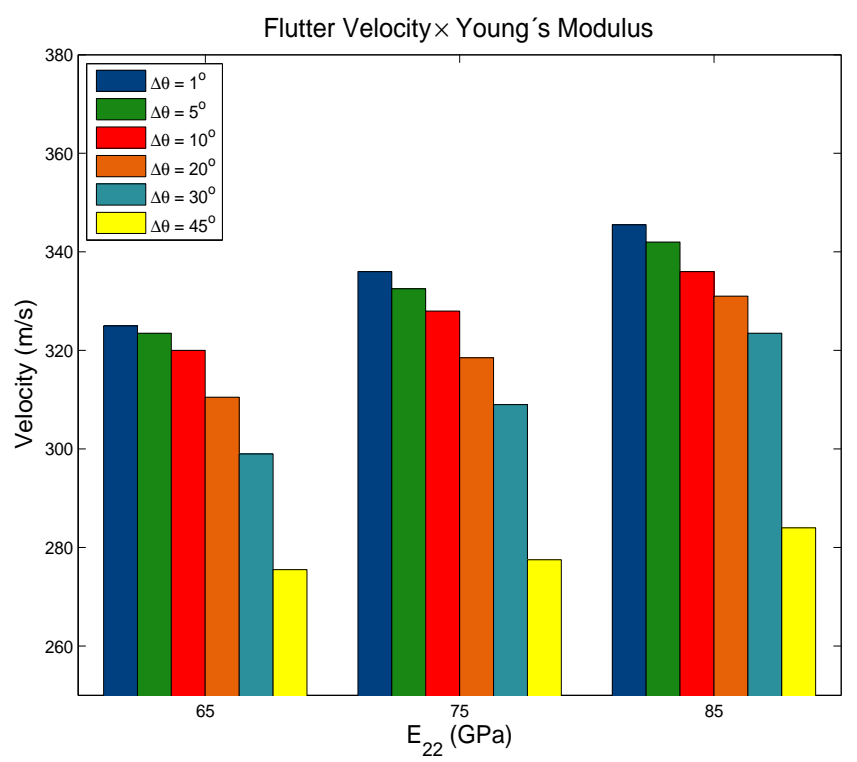

Figure 5. Flutter Velocities vs. Young's Modulus for different $\Delta \theta$ ply orientation.

As mentioned before, the same problem was already solved by a hybrid approach where deterministic (SQP) and stochastic (GA) methods were used coupled in a similar twolevel scheme (SQP + GA). In the present work, the same problem was solved by using only 
the proposed GA metaheuristics for both optimization levels (GA + GA). Table 1 presents the maximum values of flutter velocity achieved by each of the approaches, considering $E_{22}=65 \mathrm{GPa}$ and different angle variations. The approach based only on GA metaheuristics provided a better final configuration than the one provided by the hybrid method based on the deterministic method, for all angle variations. Since the GA executes a more comprehensive search on the solution domain than a deterministic method, the complete stochastic strategy achieved better results.

Table 1. Maximum Flutter Velocity (m/s) achieved by different optimization strategies.

\begin{tabular}{ccccccc}
\hline Methods & \multicolumn{7}{c}{$\Delta \theta$} \\
\hline \hline 1st lev. + 2nd lev. & $1^{\circ}$ & $5^{\circ}$ & $10^{\circ}$ & $20^{\circ}$ & $30^{\circ}$ & $45^{\circ}$ \\
\hline SQP + AG [5] & 316.0 & 314.0 & 304.0 & 299.5 & 282.0 & $-\times-$ \\
AG + AG & 325.0 & 323.5 & 320.0 & 310.5 & 299.0 & 275.5 \\
\hline
\end{tabular}

Moreover, the same problem was solved by using the proposed GA metaheuristics coupled with the local search heuristic for both optimization levels $(\mathrm{GA}+\mathrm{HJ}+\mathrm{GA}+\mathrm{HJ})$. Therefore, a comparison between the GA-based approach and the GA+HJ-based approach is presented in Table 2, now assuming only three different values for the angle variation. Table 2 shows the maximum ( $\max$ ), average (avg) and standard deviation (std) values computed from a set of 30 runs of each optimization approach and assuming different angle variations. In order to make a comparison easier, the values obtained with the GA-based approach were inserted into parenthesis next to the values obtained with the GA+HJ-based approach. The hybrid approach based on the local search provided better average values, together a standard deviation reduction, except when $\Delta \theta_{j}=45^{\circ}$. Concerning the maximum values obtained, the hybrid approach based on the local search provided better results as well, except when $\Delta \theta_{j}=1^{\circ}$.

Table 2. Maximum Flutter Velocity $(\mathrm{m} / \mathrm{s})$ achieved by the AG+HJ approach.

\begin{tabular}{cccc}
\hline Methods & \multicolumn{3}{c}{$\Delta \theta$} \\
\hline \hline 1st lev. + 2nd lev. & $1^{\circ}$ & $10^{\circ}$ & $45^{\circ}$ \\
\hline AG+HJ + AG+HJ (max) & $323.0(325.0)$ & $323.0(320.0)$ & $276.5(275.5)$ \\
AG+HJ + AG+HJ (avg) & $319.70(319.65)$ & $312.93(307.92)$ & $252.15(248.45)$ \\
AG+HJ + AG+HJ (std) & $1.97(2.48)$ & $4.96(5.24)$ & $11.11(9.29)$ \\
\hline
\end{tabular}

With the objective to highlight the superiority of the hybrid approach with local search when compared with the approach without local search, the percentage of solutions above a predetermined threshold provided by each one of the approaches was counted. It should be remarked that the percentage results were obtained based on a set of 30 independent runs performed for each one of the approaches. Table 3 shows the percentage values obtained when using the AG-based approach and the AG+HJ-based approach. The velocity values assumed as threshold for each angle variation follow: $v_{1^{\circ}}=320 \mathrm{~m} / \mathrm{s}, v_{10^{\circ}}=310 \mathrm{~m} / \mathrm{s}$ and $v_{45^{\circ}}=255$ $\mathrm{m} / \mathrm{s}$.

Based on the percentage values presented in Table 3, the superiority of the AG+HJbased approach is evident, with percentage values above $50 \%$ for two instances, $\Delta \theta=1^{\circ}$ and $\Delta \theta=10^{\circ}$. For the third instance, the approach with local search complied with the threshold almost the double, if compared with the approach without local search. 
Table 3. Optimization approaches performance, with and without local search.

\begin{tabular}{cccc}
\hline Methods & \multicolumn{3}{c}{$\Delta \theta$} \\
\hline \hline 1st lev. + 2nd lev. & $1^{\circ}$ & $10^{\circ}$ & $45^{\circ}$ \\
\hline AG+HJ + AG+HJ & $55 \%$ & $70 \%$ & $30 \%$ \\
AG + AG & $40 \%$ & $37 \%$ & $17 \%$ \\
\hline
\end{tabular}

\section{CONCLUSIONS}

A hybrid two level composite wing-box aeroelastic design optimization was presented and evaluated. The procedure is based on a real valued genetic algorithm metaheuristic used at the first level for the determining of the optimal anisotropy parameters, here defined to describe the aeroelastic composite properties of the wing-box. At the second level an integer based on a genetic algorithm technique was used to determine the composite laminate angles associated to the first level anisotropy parameters. A communication criterion, based on Tsai-Hill strength condition and on the second level objective functions, was also introduced between the two optimization levels. The results here presented have shown that this procedure can be used to find an optimal wing design, at least from the flutter analysis here considered, where the approach with a local search coupled with the genetic algorithm is an appropriate choice if compared with other strategies.

\section{Acknowledgements}

The authors gratefully acknowledge the financial support of the Brazilian Council for Scientific and Technological Development (CNPq) (Grant Proc. 483924/2009-1). 


\section{REFERENCES}

[1] Ashton J., Halpin J., Petit P., "Primer on Composite Materials Analysis". Westport: Technomic, Boston, 1969.

[2] Bisplinghoff R., Ashley H., Halfman R., "Aeroelasticity". Dover Publication, Inc., New York, 1996.

[3] Chiwiacowsky L., Campos Velho H., "Different approaches for the solution of a backward heat conduction problem". Inverse Problems in Engineering, 11(6), 471-494, 2003.

[4] Gasbarri P., Betti F., Persiani F., Saggiani G., "Static aeroelastic control of an adaptive wing". Proceedings of the 19th Congress of the International Council of the Aeronautical Sciences, 589-603, 1994.

[5] Gasbarri P., Chiwiacowsky L. D., Campos Velho H. F., "A hybrid multilevel approach for aeroelastic optimization of composite wing-box". Structural and Multidisciplinary Optimization, 39, 607-624, 2009.

[6] Goldberg D., "Genetic algorithms in search, optimization, and machine learning". Addison-Wesley Publishing Company Inc., New York, 1989.

[7] Gurdal Z., Haftka R., Hajela P., "Design and Optimization of Laminated Composite Materials". John Wiley \& Sons, New York, 1999.

[8] Hooke R., Jeeves T.A., “ "Direct Search” Solution of Numerical and Statistical Problems”. Journal of the Association for Computing Machinery (ACM), 8(2), 212-229, 1961.

[9] Kelley C. T., "Iterative Methods for Optimization, volume 18 of Frontiers in Applied Mathematics". SIAM, Philadelphia, PA, USA, 1999.

[10] Michalewicz Z., "Genetic algorithms + data structures = evolution programs". SpringerVerlag, Berlin, 1996.

[11] Mitchell M., "An introduction to genetic algorithms". The MIT Press, Cambridge, Massachusetts, 1996.

[12] Santini P., Gasbarri P.,. "Lifting surface in subsonic unsteady regime. Meccanica", International Journal of the Italian Association of Theoretical and Applied Mechanics, 34(1), 1-27, 1999.

[13] Santini P., Gasbarri P., "Structural dynamics of a cantilever wing-like anisotropic swept plate wings". Journal of Reinforced Plastic and Composite, 19(14), 1112-1146, 2000.

[14] Sobieszczanski-Sobieski J., "Two alternative ways for solving the coordination problem in multilevel optimization". Structural and Multidisciplinary Optimization, 6(4), 205215, 1993.

[15] Sobieszczanski-Sobieski J., James B., Dovi A., "Structural optimization by multilevel decomposition". AIAA Journal, 23(11), 1775-1782, 1985.

[16] Talbi E.G., "Metaheuristics: from design to implementation". Wiley, New Jersey, 2009.

[17] Walsh J., Young K., Pritchard J., Adelman H., Mantay W., "Integrated aerodynamic/dynamic/structural optimization of helicopter rotor blades using multilevel decomposition”. Technical report, NASA TP-3465, 1995.

[18] Wrenn G., Dovi A., "Multilevel decomposition approach to the preliminary sizing of a transport aircraft wing”. AIAA Journal of Aircraft, 25(7), 632-638, 1988.

[19] Zapfel G., Braune R., Bogl M., "Metaheuristic Search Concepts: A tutorial with applications to production and logistics". Springer, 2010 